\title{
SGTA associates with intracellular aggregates in neurodegenerative diseases
}

\author{
Shun Kubota ${ }^{1}$, Hiroshi Doi ${ }^{* *}$, Shigeru Koyano ${ }^{1,2}$, Kenichi Tanaka' , Hiroyasu Komiya', Atsuko Katsumoto', \\ Shingo Ikeda' ${ }^{1}$, Shunta Hashiguchi ${ }^{1}$, Haruko Nakamura', Ryoko Fukai ${ }^{1}$, Keita Takahashi ', Misako Kunii', \\ Mikiko Tada', Hideyuki Takeuchi ${ }^{1}$ and Fumiaki Tanaka ${ }^{1 *}$ (D)
}

\begin{abstract}
Intracellular aggregates are a common pathological hallmark of neurodegenerative diseases such as polyglutamine (polyQ) diseases, amyotrophic lateral sclerosis (ALS), Parkinson's disease (PD), and multiple system atrophy (MSA). Aggregates are mainly formed by aberrant disease-specific proteins and are accompanied by accumulation of other aggregate-interacting proteins. Although aggregate-interacting proteins have been considered to modulate the formation of aggregates and to be involved in molecular mechanisms of disease progression, the components of aggregate-interacting proteins remain unknown. In this study, we showed that small glutamine-rich tetratricopeptide repeat-containing protein alfa (SGTA) is an aggregate-interacting protein in neurodegenerative diseases. Immunohistochemistry showed that SGTA interacted with intracellular aggregates in Huntington disease (HD) cell models and neurons of HD model mice. We also revealed that SGTA colocalized with intracellular aggregates in postmortem brains of patients with polyQ diseases including spinocerebellar ataxia (SCA)1, SCA2, SCA3, and dentatorubral-pallidoluysian atrophy. In addition, SGTA colocalized with glial cytoplasmic inclusions in the brains of MSA patients, whereas no accumulation of SGTA was observed in neurons of PD and ALS patients. In vitro study showed that SGTA bound to polyQ aggregates through its C-terminal domain and SGTA overexpression reduced intracellular aggregates. These results suggest that SGTA may play a role in the formation of aggregates and may act as potential modifier of molecular pathological mechanisms of polyQ diseases and MSA.
\end{abstract}

Keywords: SGTA, Polyglutamine disease, Neurodegeneration, Intranuclear inclusion bodies, Multiple system atrophy

\section{Introduction}

Formation of intracellular protein aggregates, which are microscopically observed as inclusion bodies, is a common pathological feature of neurodegenerative diseases including polyglutamine (polyQ) diseases, amyotrophic lateral sclerosis (ALS), Parkinson's disease (PD), and multiple system atrophy (MSA) [1, 2]. Although the main components of protein aggregates differ in each disease,

\footnotetext{
*Correspondence: hdoi@yokohama-cu.ac.jp; ftanaka@yokohama-cu.ac.jp ${ }^{1}$ Department of Neurology and Stroke Medicine, Yokohama City University Graduate School of Medicine, 3-9 Fukuura, Kanazawa-ku, Yokohama 236-0004, Japan

Full list of author information is available at the end of the article
}

the formation process of protein aggregates is thought to share common molecular and pathological pathways.

In polyQ diseases, such as Huntington disease (HD) and several spinocerebellar ataxias (SCAs), aberrant proteins are produced by each causative gene harboring abnormally expanded polyQ tracts. These aberrant proteins change their conformation, repeat self-polymerization, and finally form insoluble aggregates [3]. PolyQ aggregates are also composed of numerous aggregateinteracting proteins. Some of these aggregate-interacting proteins are considered to be related to the process of aggregate formation and are widely associated with pathophysiology of various neurodegenerative diseases. For instance, Hdj1, an Hsp40 family chaperone protein, 
has been reported to interact with polyQ aggregates in HD model cells and animals, and suppress aggregate formation and reduce cellular toxicity in vitro [4]. It has also been reported that sequestration of Hsp70 and Sis1 into polyQ aggregates disturbs ubiquitin-proteasomemediated protein degradation and impedes intracellular protein homeostasis [5]. Ubiquilins and FET (also called TET) family proteins, which are components of polyQ aggregates [6, 7], are also related to ALS. Thus, the study of aggregate-interacting proteins could help to uncover molecular pathological mechanisms common to several neurodegenerative diseases.

Previously, we identified small glutamine-rich tetratricopeptide repeat (TPR)-containing protein alpha (SGTA) as a component of polyQ aggregates in HD model cells (unpublished data). SGTA is a cochaperone that interacts with various proteins including heat shock proteins [8]. SGTA is particularly important for intracellular protein homeostasis and associates with mislocalization of membrane proteins with hydrophobic residues, such as substrates for endoplasmic reticulum-associated degradation (ERAD) $[9,10]$. Although Sgt2, a yeast ortholog of SGTA, has been shown to interact with polyQ aggregates in yeast $[11,12]$, there are currently no studies that have demonstrated the association of SGTA with intracellular aggregates of human neurodegenerative diseases. The purpose of this study was to evaluate the localization of SGTA in cell and animal models, in postmortem brains of patients with polyQ diseases, PD, and MSA, and in the spinal cord of ALS patients.

\section{Materials and methods Antibodies}

We used the following antibodies: polyclonal anti-SGTA antibody (TA308276, 1:200 for immunocytochemistry and immunohistochemistry of human tissue; Origene Technologies, Rockville, MD), monoclonal anti-SGTA antibody (sc-374031, 1:250 for western blotting, 1:100 for immunohistochemistry of mouse tissue; Santa Cruz Biotechnology, Santa Cruz, CA), monoclonal anti-ubiquitin antibody (MAB1510, 1:250 for immunocytochemistry; Chemicon, Temecula, CA), polyclonal anti-Lamin B (M-20) antibody (sc-6217, 1:250 for western blotting; Santa Cruz Biotechnology), monoclonal anti-expanded polyQ antibody (1C2, MAB1574, 1:5000 for immunohistochemistry; Chemicon), monoclonal anti-BAG6 antibody (sc-365928, 1:250; Santa Cruz Biotechnology), monoclonal anti-phosphorylated- $\alpha$-synuclein antibody (pSyn\#64, 1:400; Wako Chemicals, Tokyo, Japan), monoclonal anti-phosphorylated TDP-43 antibody (TIP-PTDM01, 1:1000; Cosmo Bio Co, Tokyo, Japan), polyclonal anti-V5 antibody (A190-120A, 1:500; Wako Chemicals, Montgomery, TX), polyclonal anti-GFP antibody (598,
1:500; Medical and Biological Laboratories, Nagoya, Japan), HRP-conjugated anti-mouse IgG and anti-rabbit IgG antibodies (NA931 and NA934, 1:5000; GE Healthcare, Buckinghamshire, UK), and anti-mouse IgG conjugated with Alexa-546 and anti-rabbit IgG conjugated Alexa-488 antibodies (A11003 and A11034, 1:1000; Thermo Fisher Scientific, Waltham, MA).

\section{HD model cells and mice}

Neuro2a cell lines were stably transfected with truncated $\mathrm{N}$-terminal huntingtin (tNhtt), which expresses a cDNA encoding huntingtin (htt) exon 1 containing 16 or 150 CAG repeats and was fused with enhanced green fluorescent protein (EGFP) with or without nuclear translocation signals (NLS). We generated four cell types including: tNhtt-16Q-EGFP (HD16Q cells), tNhtt-150QEGFP (HD150Q cells), tNhtt-16Q-EGFP-NLS (HD16QNLS cells), and tNhtt-150Q-EGFP-NLS (HD150Q-NLS cells). These cells were induced with $1 \mu \mathrm{M}$ Ponasterone A and cultured as previously described [7]. Brain slices from two heterozygous htt exon 1 transgenic male mice of the R6/2 (145 CAG repeats) strain [Jackson code, B6CBA-TgN (HD exon1) 62] and two age-matched controls at 12 weeks of age were used for immunohistochemistry. The HD model cells and mouse brain samples were a kind gift from Dr. Nobuyuki Nukina (Doshisha University, Japan). All animal experiments conformed to the Guide for the Care and Use of Laboratory Animals.

\section{Human specimens}

Human brain specimens were obtained during postmortem examination of patients with polyQ diseases (SCA1, SCA2, SCA3, and dentatorubral-pallidoluysian atrophy [DRPLA]), ALS, PD, and MSA (Table 1). Full consent from the family was obtained at the time of autopsy, and the Hospital Human Subjects Ethics Committee approved the study. Diagnoses were based on clinical and pathological findings. Human specimens were fixed in $10 \%$ formalin for 1 to 3 weeks, embedded in paraffin, and sectioned at a thickness of $5 \mu \mathrm{m}$.

\section{Plasmid construction}

The cDNA for human SGTA was obtained from Mammalian Gene Collection cDNA clones (Invitrogen, Carlsbad, CA) and subcloned into the TOPO-pcDNA3.1/ V5-His mammalian expression vector (Invitrogen). Using this SGTA vector as a template, SGTA deletion mutants were created by PCR and subcloned into the same vector. All constructs were verified by sequencing. Hdj1 plasmid was a kind gift from Dr. Nobuyuki Nukina (Doshisha University, Japan). 
Table 1 Clinical characteristics of postmortem cases

\begin{tabular}{|c|c|c|c|c|c|}
\hline Disease & $\begin{array}{l}\text { Case } \\
\text { number }\end{array}$ & $\begin{array}{l}\text { Age at } \\
\text { death } \\
\text { (years) }\end{array}$ & $\operatorname{Sex}(M / F)$ & $\begin{array}{l}\text { Disease } \\
\text { duration } \\
\text { (months) }\end{array}$ & $\begin{array}{l}\text { SGTA- } \\
\text { reactive } \\
\text { inclusions }\end{array}$ \\
\hline SCA1 & 1 & 55 & $\mathrm{~F}$ & 144 & Positive \\
\hline SCA1 & 2 & 71 & $\mathrm{~F}$ & 492 & Positive \\
\hline SCA2 & 3 & 57 & M & 120 & Positive \\
\hline SCA2 & 4 & 48 & $\mathrm{~F}$ & 252 & Positive \\
\hline SCA3 & 5 & 40 & $\mathrm{~F}$ & 156 & Positive \\
\hline SCA3 & 6 & 51 & $F$ & 240 & Negative \\
\hline SCA3 & 7 & 36 & M & 168 & Positive \\
\hline DRPLA & 8 & 46 & $F$ & 131 & Positive \\
\hline PD & 9 & 82 & $F$ & 155 & Negative \\
\hline$P D$ & 10 & 94 & F & 324 & Negative \\
\hline$P D$ & 11 & 82 & $\mathrm{~F}$ & 66 & Negative \\
\hline PD & 12 & 58 & $\mathrm{~F}$ & 228 & Negative \\
\hline MSA & 13 & 48 & M & 85 & Positive \\
\hline MSA & 14 & 75 & M & 84 & Positive \\
\hline MSA & 15 & 65 & $\mathrm{~F}$ & 40 & Positive \\
\hline MSA & 16 & 79 & $F$ & 48 & Positive \\
\hline ALS & 17 & 69 & $\mathrm{~F}$ & 7 & Negative \\
\hline ALS & 18 & 64 & M & 36 & Negative \\
\hline ALS & 19 & 87 & M & 29 & Negative \\
\hline ALS & 20 & 61 & M & 9 & Negative \\
\hline ALS & 21 & 75 & $\mathrm{~F}$ & 7 & Negative \\
\hline ALS & 22 & 72 & M & 20 & Negative \\
\hline
\end{tabular}

$M$ male, $F$ female, SCA spinocerebellar ataxia, DRPLA dentatorubralpallidoluysian atrophy, PD Parkinson's disease, MSA multiple system atrophy, ALS amyotrophic lateral sclerosis

\section{Immunocytochemistry and immunohistochemistry}

Immunostaining was performed as reported previously. [6] In brief, HD150Q cells or HD150QNLS cells were differentiated with $5 \mathrm{mM}$ $\mathrm{N}^{6}, 2^{\prime}$-O-dibutyryladenosine- $3^{\prime}: 5^{\prime}$-cyclic monophosphate sodium salt (dbcAMP, Nacalai, Kyoto, Japan) and induced to express tNhtt-polyQ with $1 \mu \mathrm{M}$ Ponasterone A. After 2 days, immunocytochemistry was performed. The frozen mouse brain sections and paraffin-embedded human specimens were also subjected to immunohistochemistry as described previously [13]. Briefly, frozen mouse brain sections were washed twice with phosphate-buffered saline (PBS), fixed for $30 \mathrm{~min}$ with $100 \%$ methanol, washed three times PBS, blocked for $1 \mathrm{~h}$ with TBST containing $2 \%$ non-fat dried milk, and incubated overnight at $4{ }^{\circ} \mathrm{C}$ with primary antibodies. Then, the sections were washed three times with TBST and incubated with secondary antibody for $2 \mathrm{~h}$, and detected using the Vectastain ABC kit (Vector Laboratories, Burlingame, CA). Human brain sections were autoclaved at $121{ }^{\circ} \mathrm{C}$ for $10 \mathrm{~min}$, incubated in 100\% formic acid for $5 \mathrm{~min}$, and incubated with $1 \%$ hydrogen peroxidase for $15 \mathrm{~min}$. The sections were then blocked for $1 \mathrm{~h}$ with TBST containing $7 \%$ goat serum, and then immunostained with primary antibodies followed by visualization using the Vectastain $\mathrm{ABC}$ kit. We confirmed the existence of polyQ inclusions with anti-expanded polyQ (1C2) antibody in polyQ disease cases (SCA1, SCA2, SCA3 and DRPLA), $\alpha$-synuclein aggregates with anti-phosphorylated $\alpha$-synuclein antibody in PD and MSA cases, and neuronal inclusions with anti-phosphorylated TDP-43 antibody in ALS cases. Then, SGTA immunoreactivity was investigated in relation to the localization of these pathological hallmarks in each case using double immunofluorescence staining visualized by a confocal laser scanning microscope (FV1000D, Olympus, Tokyo, Japan).

\section{Preparation of soluble and insoluble fractions from cell models}

After HD model cells were differentiated and induced for $24 \mathrm{~h}$, they were washed twice in ice cold PBS and harvested in lysis buffer (50 mM Tris- $\mathrm{HCl}$ [pH 7.5], $150 \mathrm{mM}$ $\mathrm{NaCl}, 1 \%$ Triton $\mathrm{X}-100,0.1 \%$ SDS, 1 mM EDTA, 0.5\% sodium deoxycholate, and complete protease inhibitor cocktail [Roche, Basel, Switzerland]). Cell lysates were centrifuged at $10,000 \times g$ for $30 \mathrm{~min}$ and the resulting pellets were resuspended in lysis buffer. To prevent contamination of the soluble components by pellets, this process was repeated three times. The first supernatants and the final pellets were regarded as the soluble fractions and the insoluble fractions, respectively. The final pellets were then dissolved in urea buffer $(30 \mathrm{mM}$ Tris $\mathrm{pH}$ 8.5, $7 \mathrm{M}$ urea and $2 \mathrm{M}$ thiourea, 4\% 3-[(3-Cholamidoproryl) dimenthylammonio]-1-propanesulfonate (CHAPS, 07957-51, Nacalai)). Each fraction was subjected to western blot analysis.

\section{Deletion assay}

HD 150Q cells were transfected with the SGTA construct containing each deletion mutant using Lipofectamine LTX (Thermo Fisher Scientific) according to the manufacturer's protocol. Twenty-four hours after transfection, these cells were differentiated and expression of tNhtt150Q-GFP was induced. After another $24 \mathrm{~h}$, immunocytochemistry was performed using anti-V5 antibody. Immunostaining was performed as reported previously [7].

\section{Filter trap assay}

A filter trap assay was performed using a dot blot apparatus (SCIE-PLAS, Cambridge, UK) according to the method of Wanker et al. [14]. HD150Q cells were transfected with an expression vector construct for SGTA, LacZ or Hdj1. Twenty-four hours after transfection, cells were harvested and each cell lysate was filtered 
with a 0.2- $\mu \mathrm{m}$-pore cellulose acetate membrane. After washing with PBS twice, membranes were analyzed by immunoblotting.

\section{Statistical analysis}

For deletion assay and the filter trap assay, numerical comparison was performed with the Student's t-test. Differences were considered significant at $P<0.05$.

\section{Results}

SGTA colocalized with polyQ aggregates in HD model cells during aggregate formation

First, we confirmed whether SGTA colocalizes with intracellular polyQ aggregates in HD model cells (HD150Q cells and HD150Q-NLS cells). Expanded polyQ-containing protein tNhtt-150Q or tNhtt-150Q-NLS formed aggregates in the cytoplasm or nucleus of HD model cells, respectively. In these cells, endogenous SGTA colocalized with cytoplasmic aggregates or intranuclear aggregates formed by the expanded polyQ-containing protein (Fig. 1a). In HD16Q and HD16Q-NLS cells, which were transfected with tNhtt-16Q or tNhtt-16Q-NLS, respectively, SGTA was diffusely distributed throughout the cytoplasm (Additional file 1, Fig. S1).

Next, we assessed SGTA biochemical properties in wild type Neuro2a, HD16Q, and HD150Q cells. In wild type and HD16Q cell lysates, SGTA was detected in the total and soluble fraction but not in the insoluble fraction, whereas SGTA was detected in both the soluble and insoluble fractions of HD150Q cells (Fig. 1b). These results suggest that SGTA shifted from soluble to insoluble upon aggregate formation.

\section{SGTA associated with aggregates in HD model mice}

Next, we investigated whether SGTA colocalizes with polyQ aggregates in R6/2 mice using immunohistochemistry. SGTA associated with neuronal intranuclear inclusions (NIIs) in the CA1 region of mouse brains (Fig. 2a). This colocalization was confirmed by double immunofluorescence staining with antibodies against SGTA and ubiquitin. In control mouse brain, SGTA was diffusely distributed throughout the cytoplasm of neurons.

\section{SGTA colocalized with neuronal intranuclear inclusions in patients with polyQ diseases}

Next, we examined whether SGTA associates with NIIs in postmortem brains of patients with polyQ diseases. We performed immunohistochemistry on postmortem brain sections from eight patients with SCA1 $(n=2)$, SCA2 $(\mathrm{n}=2)$, SCA3 $(\mathrm{n}=3)$, and DRPLA $(\mathrm{n}=1)$. Immunohistochemistry showed that SGTA localized to NIIs in the pontine nucleus of brains from all of the polyQ disease patients except one patient with SCA3 (case 6 in Table 1;
Fig. 2b). Furthermore, double immunofluorescence staining using anti-SGTA antibody and anti-expanded polyQ antibody (1C2) was performed. In the brain of a patient with SCA1, SGTA colocalized with NIIs in the pontine nucleus (Fig. 2c), indicating an association of SGTA with polyQ aggregates. SGTA modulates intracellular protein quality control in cooperation with the BCL2-associated athanogene 6 (BAG6) complex, therefore we also examined whether BAG6 associates with NIIs in postmortem brains of patients with polyQ diseases. BAG6 was not detected in aggregates in HD model mouse brains and postmortem polyQ disease brains (Additional file 1, Fig. S2).

\section{SGTA colocalized with glial cytoplasmic inclusions in patients with MSA}

We also performed immunohistochemistry on tissue from four PD patients and four MSA patients, and on spinal cord from six ALS patients. In the MSA brains, SGTA was diffusely distributed in the cytoplasm of neurons. Strong SGTA staining was also detected in the cytoplasm of glial cells, showing similar distribution to glial cytoplasmic inclusions (GCIs), a pathological hallmark of MSA (Fig. 3a). Double immunofluorescence staining using anti-SGTA antibody and anti-phosphorylated $\alpha$-synuclein antibody revealed that SGTA colocalized with phosphorylated $\alpha$-synuclein in GCIs (Fig. 3b). In brains of PD patients, SGTA was diffusely detected in the cytoplasm of neurons (Fig. 3a). Double immunofluorescence staining with anti-phosphorylated $\alpha$-synuclein and anti-SGTA antibodies showed that SGTA did not colocalize with phosphorylated $\alpha$-synuclein-reactive portions of Lewy bodies. However, in the cases with some mature Lewy bodies with halo structure, SGTA antibody weakly reacted with the core where no phosphorylated $\alpha$-synuclein reactivity was detected (Additional file 1, Fig. S3). In spinal cords of ALS patients, SGTA was diffusely distributed in the cytoplasm of neurons (Fig. 3a), and double immunofluorescence staining with antiphosphorylated TDP-43 antibody revealed that neuronal cytoplasmic inclusions of ALS were not stained by SGTA antibody (Additional file 1, Fig. S4).

\section{SGTA interacts with polyQ aggregates through its C-terminal domain}

Next, to identify the SGTA domain that interacts with aggregates, HD150Q cells were transfected with expression plasmids encoding V5-tagged full-length SGTA and three types of SGTA deletion mutants (Fig. 4a). Immunocytochemistry showed that full-length SGTA and the SGTA C-terminal domain colocalized with polyQ aggregates (Fig. 4b). Deletion mutants of SGTA protein containing the $\mathrm{N}$-terminal or TPR domains were diffusely 
a

HD150Q

HD150Q-NLS
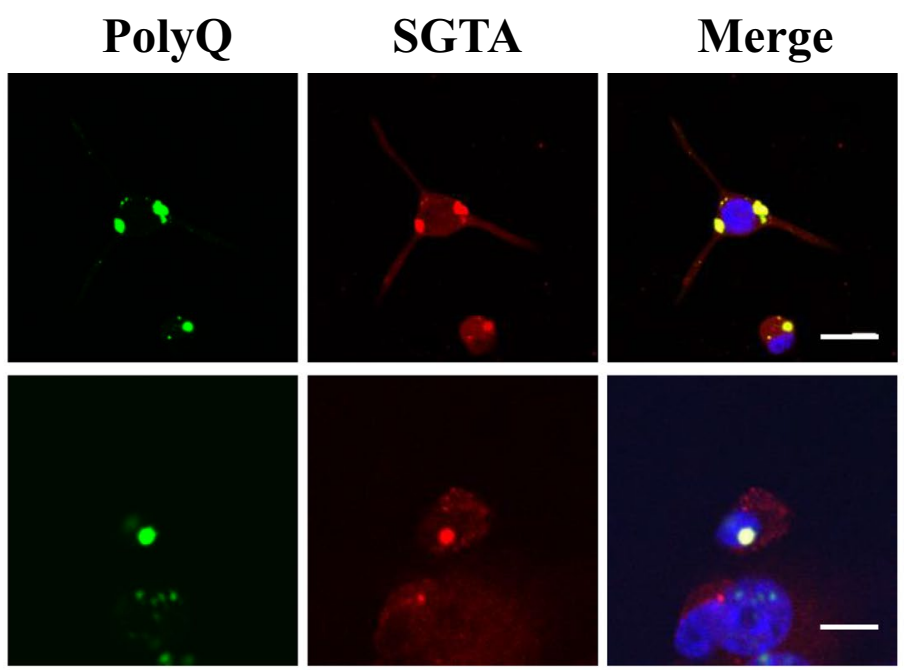

b

Neuro2a WT,

\section{Total soluble insoluble}

HD16Q cell, HD150Q cell
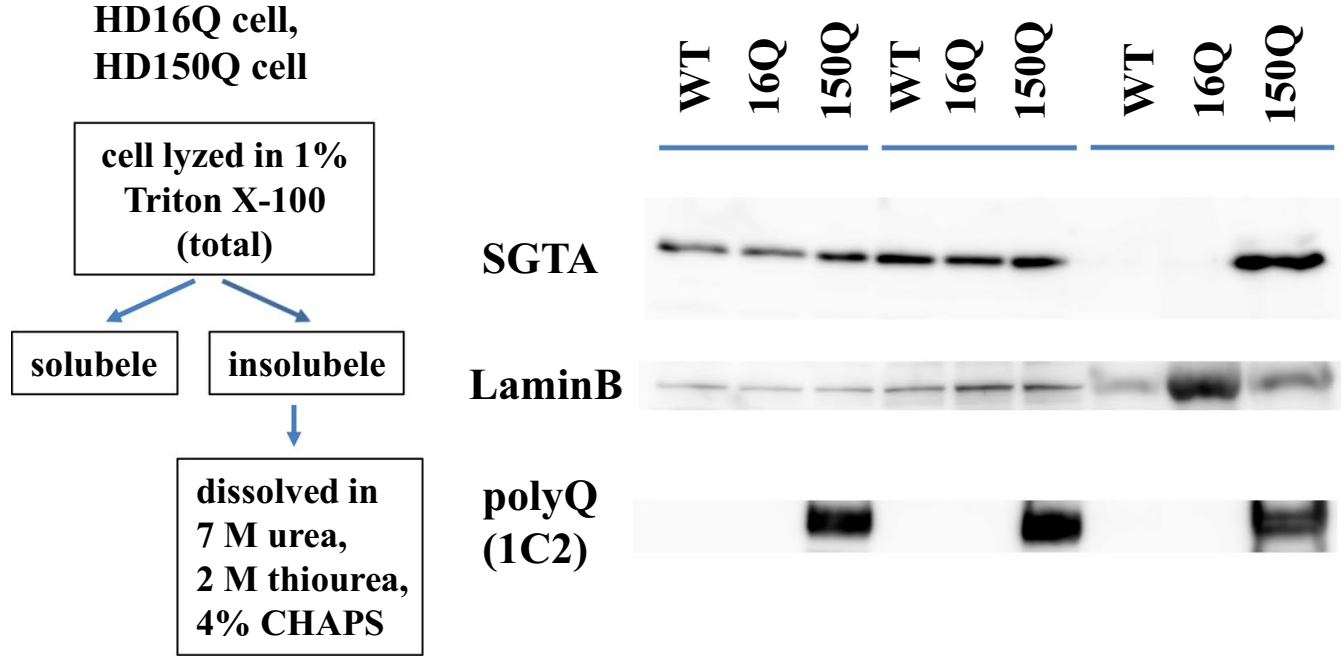

SGTA

\section{LaminB}

polyQ

(1C2)
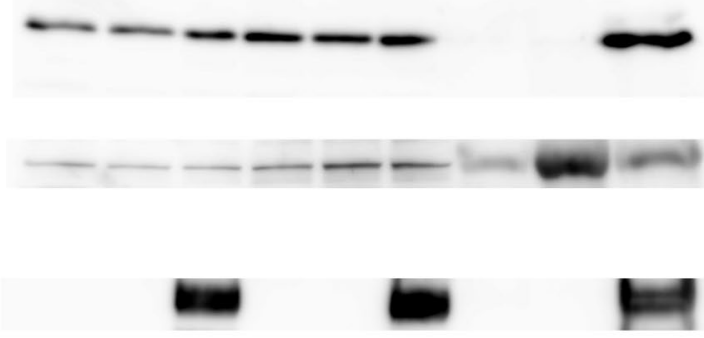

Fig. 1 SGTA colocalized with polyQ aggregates in HD model cells. a Immunocytochemistry of Huntington disease (HD) model cells, HD150Q cells or HD 150QNLS cells. PolyQ aggregate formation was induced in these cells. Endogenous small glutamine-rich tetratricopeptide repeat (TPR)-containing protein alpha (SGTA) was labeled with anti-SGTA antibody (secondary antibody: Alexa Fluor 546). The nuclei were stained with 4',6-diamidino-2-phenylindole (DAPI). Scale bar $=20 \mu \mathrm{m}$. b Cell lysates of Neuro2a wild type (WT), HD16Q, and HD150Q cells were fractionated into soluble and insoluble fractions. Immunoblotting with anti-SGTA, anti-LaminB and anti-1C2 antibodies was performed

expressed in cytoplasm without aggregate formation. We counted the number of SGTA-positive aggregates among the polyQ aggregates in cells transfected with deletion mutants of SGTA. C-terminal domain of SGTA-transfected cells showed the most abundant SGTA-positive polyQ aggregates, suggesting that the $\mathrm{C}$-terminal domain of SGTA interacts with polyQ aggregates (Fig. 4b). Next, HD16Q and HD150Q cell lysates were immunoprecipitated with anti-GFP antibody and subjected to immunoblot analysis with anti-SGTA antibody. We did not observe interaction of tNhtt-polyQ and SGTA (Additional file 1, Fig. S5).

\section{SGTA overexpression reduced polyQ aggregates in HD model cells}

Finally, we assessed the effect of overexpression of SGTA on intracellular aggregate formation. HD150Q cells were transfected with SGTA, LacZ, or Hdj1 expression plasmid vectors. The levels of aggregates in SGTA-transfected cells were significantly lower than 


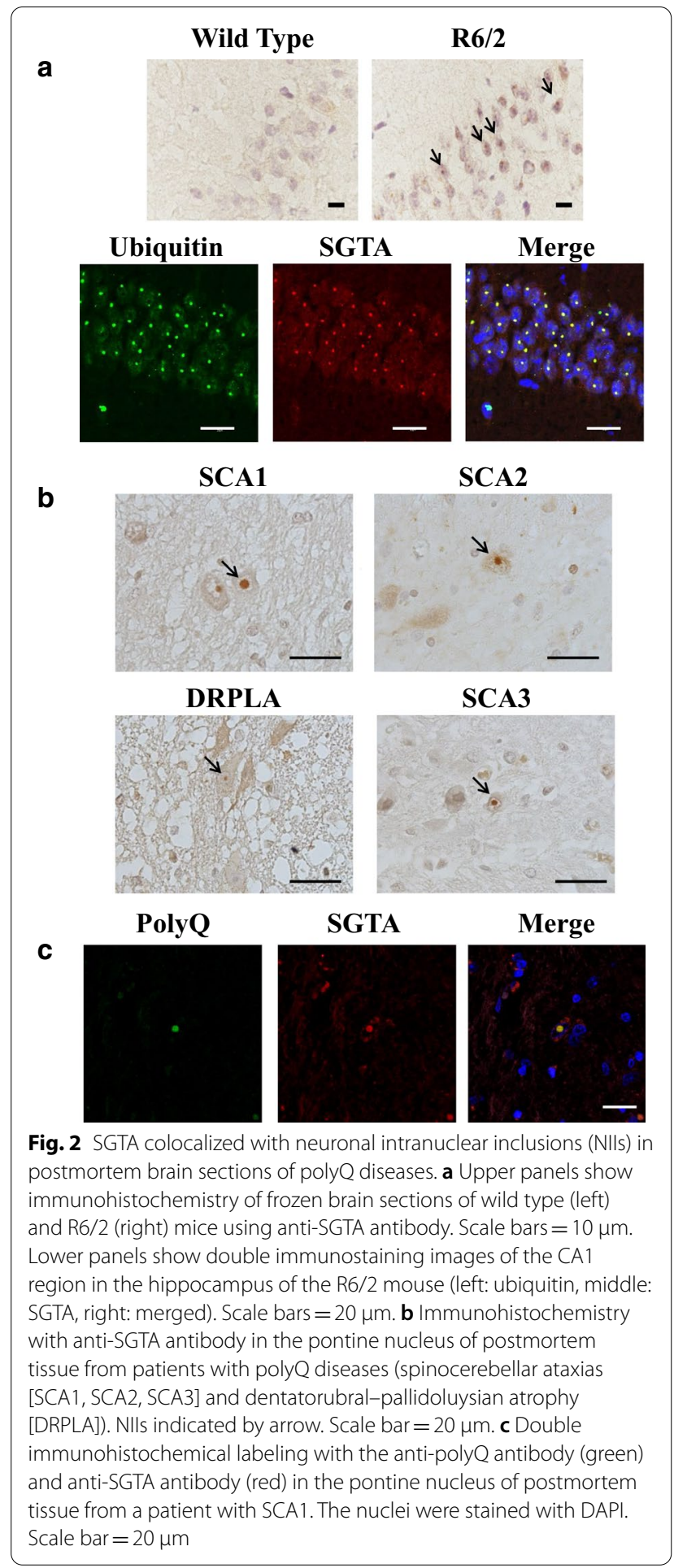

those in LacZ-transfected cells (Fig. 4c). Hdj1, a heat shock protein family member, was used as a positive control for aggregate formation suppression [4] and also demonstrated reduced aggregate formation in HD

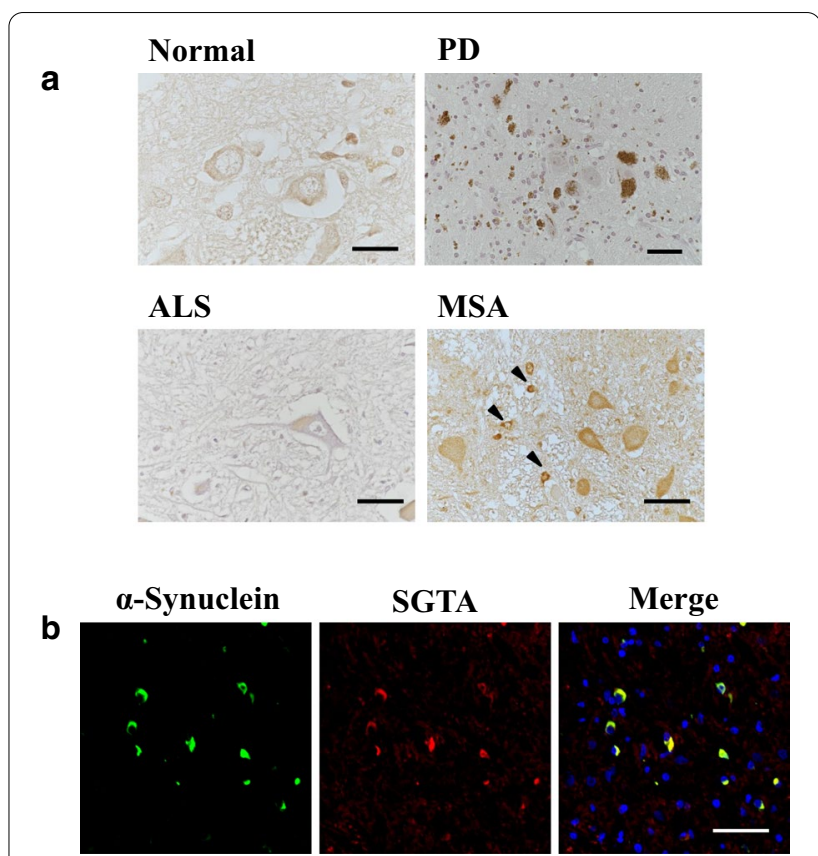

Fig. 3 SGTA interacted with glial cytoplasmic inclusions (GCls) in multiple system atrophy (MSA). a Immunohistochemistry of postmortem tissue with anti-SGTA antibody in the substantia nigra of PD patients, in the medulla of MSA patients, in the spinal cord of ALS patients, and in the pontine nucleus of control patients. Control patients: scale bar $=20 \mu \mathrm{m}$, others: scale bar $=50 \mu \mathrm{m}$. Note that intense staining observed in the substantia nigra of PD patients represents the melanin pigments. Arrow heads indicate SGTA staining of GCls in oligodendrocytes. b Double fluorescence immunohistochemistry labeling with anti-phosphorylated a-synuclein antibody (green) and anti-SGTA antibody (red) in the medulla of a patient with MSA. Scale bar $=50 \mu \mathrm{m}$

model cells. The densitometric analysis showed levels of aggregates in SGTA- and Hdj1-overexpressing cells were $77.0 \pm 5.3 \%, P=0.004$ and $70.3 \pm 7.0 \%, P=0.004$ (mean \pm SEM; $n=6$ in each case) lower, respectively, than levels in LacZ-transfected cells. We also counted and compared the number of polyQ aggregate-positive cells among the cells transfected with each plasmid vector (LacZ, SGTA or Hdj1). In SGTA-transfected cells, the percentage of aggregate-positive cells did not significantly change compared with the percentage in LacZ-transfected cells (Additional file 1, Fig. S6A). We further performed flow cytometric analysis using HD150Q cells treated in the same manner as above and the results disclosed two GFP fluorescent peaks (weak fluorescence and strong fluorescence; Additional file 1, Fig. S6B and S6C). SGTA and Hdj1 both reduced the weak fluorescent peaks (red arrows, Additional file 1, Fig. S6B and S6C), whereas they did not affect the strong fluorescent peaks. 
a

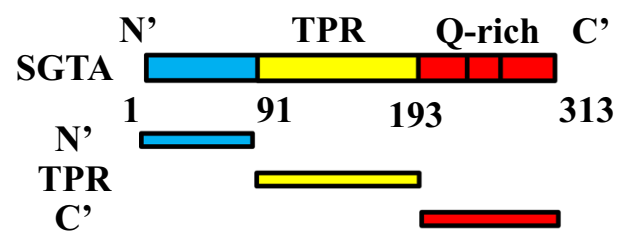

C

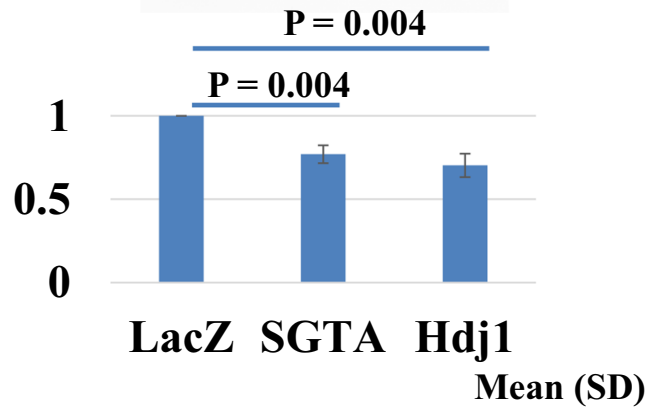

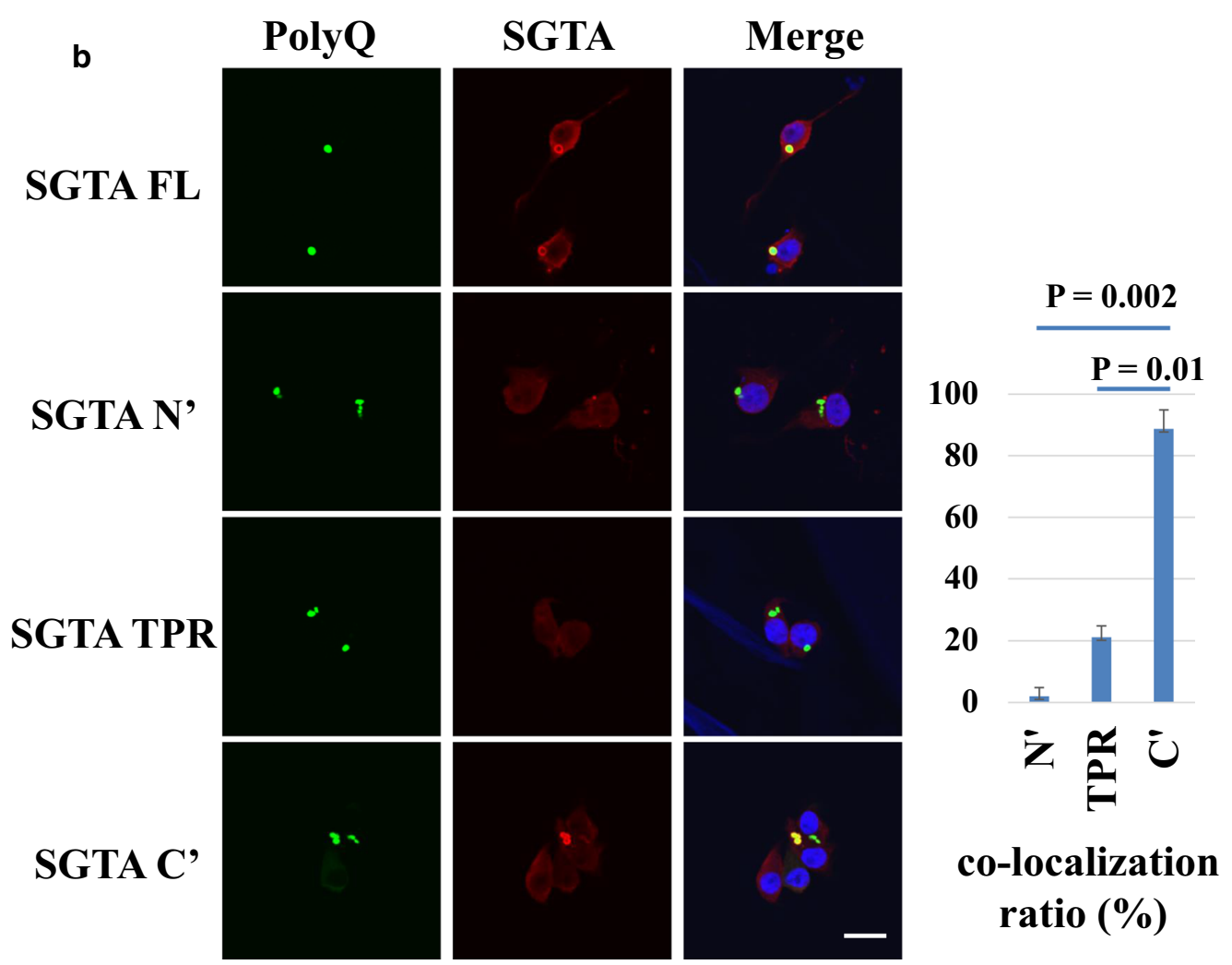

Fig. 4 C-terminal domain of SGTA interacted with aggregates in HD model cells. a Schema of the full-length SGTA and deletion mutant constructs. b Immunocytochemistry of HD150Q cells transfected with expression vector of full-length SGTA or deletion mutants. Each expression protein was tagged with V5 protein and labeled with anti-V5 antibody. Scale bar $=20 \mu \mathrm{m}$. Ratio of SGTA colocalization with polyQ aggregates is also shown. c Filter trap assay for comparing aggregate formation in SGTA-, LacZ- (negative control), and Hdj1- (positive control) overexpressing cells. HD150 cells were transfected with each vector and cell lysates were subjected to filter trap assay. Experiments were performed three times and statistical significance was assessed by Student's T-test

\section{Discussion}

In this study, we showed that SGTA is a component of polyQ aggregates. Immunostaining demonstrated that SGTA colocalized with polyQ aggregates in HD model cells and HD model mice. SGTA also associated with NIIs in patients with polyQ diseases including SCA1, SCA2, SCA3, and DRPLA. Additionally, SGTA interacted with
GCIs in postmortem tissue of patients with MSA. SGTA overexpression enhanced solubility of polyQ aggregates in HD model cells.

SGTA, originally identified as an interacting partner for nonstructural protein NS1 of parvovirus H-1 [15], has been shown to interact with the C-terminal of Hsc70 by TPR domains [16], and also with the synaptic vesicle 
cysteine string protein (CSP) to form a stable trimeric complex that functions as a chaperone machine [17]. Additional roles of SGTA have been reported, such as in the regulation of steroid hormone receptors [18, 19] and modulation of the protein quality control system by working with the BAG6 complex $[9,10]$. Depletion of SGTA causes accumulation of BAG6-dependent ERAD substrates, indicating that SGTA assists BAG6 functions to prevent the formation of nondegradable protein aggregates by ERAD [9]. In contrast, SGTA has also been reported to antagonize the actions of BAG6 and delay the proteasomal degradation of mislocalized membrane proteins (MLPs) by promoting the deubiquitination of MLPs [20-22]. Possible involvement of SGTA in neurodegeneration has been suggested based on the observation that the SGTA ortholog binds with intracellular A $\beta$ in a C. elegans model expressing a chimeric signal peptide/human A $\beta 1-42$ [23]. In addition, CSP, the binding partner of SGTA, has been shown to bind the huntingtin fragment with an expanded polyQ region [24]. Considering these findings in disease models, and the perturbation of cellular protein homeostasis and accumulation of aberrant proteins as important factors in pathophysiology of neurodegenerative diseases $[25,26]$, SGTA may be involved in the pathogenesis of neurodegenerative diseases. To the best of our knowledge, this study provides the first evidence that SGTA is associated with intracellular aggregates in postmortem brains of individuals with polyQ diseases (SCA1, SCA2, SCA3 and DRPLA) and MSA.

SGTA has been reported to associate with the hydrophobic domain of membrane proteins through its C-terminal domain [27, 28], and to cooperate with their binding partners to maintain cytosolic protein quality control [9]. In this study, immunohistochemical analysis indicated that SGTA interacts with polyQ aggregates through the C-terminal domain. This finding is consistent with previous studies because expanded polyQ tracts also have hydrophobic properties [29]. Immunoprecipitation of HD16Q and HD150Q cell lysates with anti-GFP antibody failed to reveal interaction with SGTA (Additional file 1, Fig. S5), presumably because anti-GFP antibody only precipitates soluble monomers or oligomers of tNhtt-polyQ. Thus, our results suggest that SGTA interacts with tNhtt-polyQ after the formation of insoluble aggregates.

We also showed that SGTA overexpression enhanced solubility of intracellular aggregates in HD model cells, suggesting that SGTA alters the properties of aberrant polyQ proteins (Fig. 4c). Flow cytometric analysis indicated that SGTA and Hdj1 affected the size distributions of polyQ aggregates in a similar fashion (Additional file 1, Fig. S6B and S6C). Both proteins reduced only small and presumably insoluble polyQ aggregates (i.e. weak fluorescent signals), but not large and matured poly Q aggregates (i.e. strong fluorescent signals), which may account for the discrepant results between filter trap assay (Fig. 4c) and fluorescent microscopic analysis, mainly reflecting the status of large aggregates (Additional file 1, Fig. S6A).

BAG6 was undetected in aggregates of HD model mouse brains and postmortem polyQ disease brains, suggesting that SGTA may affect polyQ aggregates using alternative pathways distinct from BAG6-mediated pathways.

SGTA also colocalized with GCIs in oligodendrocytes of MSA brains (Fig. 3), but not with phosphorylated $\alpha$-synuclein-positive portions of Lewy bodies in neurons of PD brains (Additional file 1, Fig. S3). Although both GCIs and Lewy bodies are mainly composed of $\alpha$-synuclein, these two strains of aggregates have different characteristics, such as conformation and cell toxicity [30]. It is also possible that the accumulation process and protein processing pathway of $\alpha$-synuclein might be different between neurons and oligodendrocytes. The findings suggest that SGTA may be involved in pathophysiological mechanisms for various polyQ diseases and MSA, but not necessarily for all neurodegenerative diseases including PD and ALS. Further study is required to clarify the role of SGTA in neurodegenerative processes.

In summary, we showed that SGTA is an aggregateinteracting protein in polyQ diseases and MSA. SGTA was found in polyQ-positive NIIs and $\alpha$-synucleinpositive GCIs. These findings, together with the known role of SGTA in intracellular protein homeostasis, suggest that SGTA may play an essential role in the aggregate accumulation process in these diseases. However, further study is needed to elucidate the role of SGTA in pathophysiological mechanisms of neurodegenerative diseases.

\section{Abbreviations}

ALS: Amyotrophic lateral sclerosis; BAG6: BCL2-associated athanogene 6; DAPI: 4',6-Diamidino-2-phenylindole; CSP: Cysteine string protein; dbc-AMP: $\mathrm{N}^{6}, 2^{\prime}$-O-dibutyryladenosine-3':5'-cyclic monophosphate sodium salt; DRPLA: Dentatorubral-pallidoluysian atrophy; EGFP: Enhanced green fluorescent protein; ERAD: Endoplasmic reticulum-associated degradation; GCls: Glial cytoplasmic inclusions; HD: Huntington disease; MLP: Mislocalized membrane proteins; MSA: Multiple system atrophy; NIIs: Neuronal intranuclear inclusions; NLS: Nuclear translocation signals; PBS: Phosphate-buffered saline; PD: Parkinson's disease; PolyQ: Polyglutamine; SCA: Spinocerebellar ataxia; SGTA: Small glutamine-rich tetratricopeptide repeat-containing protein alpha; $\mathrm{tNhtt:}$ Truncated N-terminal huntingtin; TPR: Tetratricopeptide repeat.

\section{Supplementary Information}

The online version contains supplementary material available at https://doi. org/10.1186/s13041-021-00770-1.

Additional file 1: Figure S1. Distribution of SGTA in HD16Q and HD16QNLS cells. Figure S2. BAG6 in brains of an HD model mouse and human polyglutamine diseases. Figure S3. SGTA in PD brain. Figure S4. SGTA 
in motor neurons in ALS spinal cords. Figure S5. Immunoprecipitation of tNhtt-polyQ proteins.Figure S6. HD150Q cells transfected with LacZ, SGTA, or Hdj1.

Additional file 2: Supplementary materials and methods, supplementary figure legends and supplementary reference.

\section{Acknowledgements}

We deeply indebted Prof. Nobuyuki Nukina for providing HD cell models and R6/2 mouse brain samples. We thank Lesley McCollum, PhD, from Edanz Group (https://en-author-services.edanz.com/ac) for editing a draft of this manuscript.

\section{Authors' contributions}

$\mathrm{HD}$, FT, and HT designed the study. SK, SI, SH and HN performed the experiments. SK and MT provided advice for the pathological experiments. RF, MK, $\mathrm{KT}$ and $\mathrm{KT}$ analyzed the data and interpreted the results. YK and AK analyzed flow cytometry data. SK. and HD wrote the main manuscript and prepared the figures. HT and FT revised the manuscript and provided conceptual advice. FT supervised the project. All authors of this paper have read and approved the final version submitted.

\section{Funding}

This work was supported by Grants-in-Aid for Scientific Research (H.D.: 18K07503, K.Tan.: 18K07504, M.T.: 19K07845, H.T:: 18K07531, F.T.: 18K07532); Grants-in-Aid for Young Scientists (R.F:: 20K16581, M.K.: 19K17014) from the Japan Society for the Promotion of Science; a Health and Labor Sciences Research Grant from the Ministry of Health, Labour and Welfare, Japan (F.T.: \#201711060A); the Takeda Science Foundation (H.D.); and a Grant for Strategic Research Promotion from Yokohama City University (F.T.: \#SK2804).

\section{Availability of data and materials}

The datasets used during the current study are available from the corresponding author on reasonable request.

\section{Declarations}

\section{Ethics approval and consent to participate}

Experimental protocols were approved by the Institutional Review Boards of Yokohama City University (A180900002) and conformed to the provisions of the Declaration of Helsinki. All experiments were performed in accordance with institutional guidelines.

\section{Consent for publication}

Written informed consent was obtained from the family for publication of this article and accompanying images.

\section{Competing interests}

The authors declare that they have no competing interests.

\section{Author details}

${ }^{1}$ Department of Neurology and Stroke Medicine, Yokohama City University Graduate School of Medicine, 3-9 Fukuura, Kanazawa-ku, Yokohama 236-0004, Japan. ${ }^{2}$ Department of Neurology, Yokohama Minami Kyosai Hospital, 1-21-1 Mutuurahigashi, Kanazawa-ku, Yokohama 236-0037, Japan.

Received: 25 August 2020 Accepted: 15 March 2021

Published online: 23 March 2021

\section{References}

1. Soto C, Pritzkow S. Protein misfolding, aggregation, and conformational strains in neurodegenerative diseases. Nat Neurosci. 2018;21:1332-40.

2. Takalo M, Salminen A, Soininen H, Hiltunen M, Haapasalo A. Protein aggregation and degradation mechanisms in neurodegenerative diseases. Am J Neurodegener Dis. 2013:2:1-14.

3. Ross CA, Poirier MA. Protein aggregation and neurodegenerative disease. Nat Med. 2004;10:510-7.
4. Jana NR, Tanaka M, Wang G, Nukina N. Polyglutamine length-dependent interaction of $\mathrm{Hsp} 40$ and $\mathrm{Hsp} 70$ family chaperones with truncated $\mathrm{N}$-terminal huntingtin: their role in suppression of aggregation and celIular toxicity. Hum Mol Genet. 2000;9:2009-18.

5. Park S-H, Kukushkin Y, Gupta R, Chen T, Konagai A, Hipp MS, et al. PolyQ proteins interfere with nuclear degradation of cytosolic proteins by sequestering the Sis1p chaperone. Cell. 2013;154:134-45.

6. Doi H, Mitsui K, Kurosawa M, Machida Y, Kuroiwa Y, Nukina N. Identification of ubiquitin-interacting proteins in purified polyglutamine aggregates. FEBS Lett. 2004;571:171-6.

7. Doi H, Okamura K, Bauer PO, Furukawa Y, Shimizu H, Kurosawa M, et al. RNA-binding protein TLS is a major nuclear aggregate-interacting protein in huntingtin exon 1 with expanded polyglutamine-expressing cells. J Biol Chem. 2008;283:6489-500.

8. Philp LK, Butler MS, Hickey TE, Butler LM, Tilley WD, Day TK. SGTA: a new player in the molecular co-chaperone game. Horm Cancer. 2013;4:343-57.

9. Xu Y, Cai M, Yang Y, Huang L, Ye Y. SGTA recognizes a noncanonical ubiquitin-like domain in the Bag6-Ubl4A-Trc35 complex to promote endoplasmic reticulum-associated degradation. Cell Rep. 2012;2:1633-44.

10. Leznicki P, High S. SGTA antagonizes BAG6-mediated protein triage. Proc Natl Acad Sci USA. 2012;109:19214-9.

11. Wang $Y$, Meriin $A B$, Costello CE, Sherman MY. Characterization of proteins associated with polyglutamine aggregates: a novel approach towards isolation of aggregates from protein conformation disorders. Prion. 2007;1:128-35.

12. Wear MP, Kryndushkin D, O'Meally R, Sonnenberg JL, Cole RN, Shewmaker FP. Proteins with intrinsically disordered domains are preferentially recruited to polyglutamine aggregates. PLoS ONE. 2015;10:e0136362.

13. Doi H, Koyano S, Suzuki Y, Nukina N, Kuroiwa Y. The RNA-binding protein FUS/TLS is a common aggregate-interacting protein in polyglutamine diseases. Neurosci Res. 2010;66:131-3.

14. Wanker EE, Scherzinger E, Heiser V, Sittler A, Eickhoff H, Lehrach H. Membrane filter assay for detection of amyloid-like polyglutamine-containing protein aggregates. Methods in enzymology. London: Academic Press; 1999. p. 375-86.

15. Cziepluch C, Kordes E, Poirey R, Grewenig A, Rommelaere J, Jauniaux JC. Identification of a novel cellular TPR-containing protein, SGT, that interacts with the nonstructural protein NS1 of parvovirus $\mathrm{H}-1$. J Virol. 1998;72:4149-56.

16. Wu S-J, Liu F-H, Hu S-M, Wang C. Different combinations of the heatshock cognate protein 70 (hsc70) C-terminal functional groups are used to interact with distinct tetratricopeptide repeat-containing proteins. Biochem J. 2001;359:419-26.

17. Tobaben S, Thakur P, Fernández-Chacón R, Südhof TC, Rettig J, Stahl B. A trimeric protein complex functions as a synaptic chaperone machine. Neuron. 2001;31:987-99.

18. Schantl JA, Roza M, De JONGAP, Strous GJ. Small glutamine-rich tetratricopeptide repeat-containing protein (SGT) interacts with the ubiquitindependent endocytosis (UbE) motif of the growth hormone receptor. Biochem J. 2003;373:855-63.

19. Buchanan G, Ricciardelli C, Harris JM, Prescott J, Yu ZC-L, Jia L, et al. Control of androgen receptor signaling in prostate cancer by the cochaperone small glutamine-rich tetratricopeptide repeat containing protein a. Cancer Res. 2007;67:10087-96.

20. Wunderley L, Leznicki P, Payapilly A, High S. SGTA regulates the cytosolic quality control of hydrophobic substrates. J Cell Sci. 2014;127:4728-39.

21. Payapilly A, High S. BAG6 regulates the quality control of a polytopic ERAD substrate. J Cell Sci. 2014;127:2898-909.

22. Leznicki P, Korac-Prlic J, Kliza K, Husnjak K, Nyathi Y, Dikic I, et al. Binding of SGTA to Rpn13 selectively modulates protein quality control. J Cell Sci. 2015;128:3187-96.

23. Fonte V, Kapulkin WJ, Taft A, Fluet A, Friedman D, Link CD. Interaction of intracellular $\beta$ amyloid peptide with chaperone proteins. Proc Natl Acad Sci USA. 2002;99:9439-44.

24. Miller LC, Swayne LA, Chen L, Feng Z-P, Wacker JL, Muchowski PJ, et al. Cysteine string protein (CSP) inhibition of N-type calcium channels is blocked by mutant Huntingtin. J Biol Chem. 2003;278:53072-81.

25. Takahashi T, Katada S, Onodera O. Polyglutamine diseases: where does toxicity come from? What is toxicity? Where are we going? J Mol Cell Biol. 2010;2:180-91. 
26. Williams AJ, Paulson HL. Polyglutamine neurodegeneration: protein misfolding revisited. Trends Neurosci. 2008;31:521-8.

27. Wang F, Brown EC, Mak G, Zhuang J, Denic V. A chaperone cascade sorts proteins for posttranslational membrane insertion into the endoplasmic reticulum. Mol Cell. 2010;40:159-71.

28. Martínez-Lumbreras S, Krysztofinska EM, Thapaliya A, Spilotros A, MatakVinkovic D, Salvadori E, et al. Structural complexity of the co-chaperone SGTA: a conserved C-terminal region is implicated in dimerization and substrate quality control. BMC Biol. 2018;16:76.

29. Burke MG, Woscholski R, Yaliraki SN. Differential hydrophobicity drives self-assembly in Huntington's disease. Proc Natl Acad Sci USA. 2003;100:13928-33.
30. Peng C, Gathagan RJ, Covell DJ, Medellin C, Stieber A, Robinson JL, et al. Cellular milieu imparts distinct pathological a-synuclein strains in a-synucleinopathies. Nature. 2018;557:558-63.

\section{Publisher's Note}

Springer Nature remains neutral with regard to jurisdictional claims in published maps and institutional affiliations.
Ready to submit your research? Choose BMC and benefit from:

- fast, convenient online submission

- thorough peer review by experienced researchers in your field

- rapid publication on acceptance

- support for research data, including large and complex data types

- gold Open Access which fosters wider collaboration and increased citations

- maximum visibility for your research: over $100 \mathrm{M}$ website views per year

At BMC, research is always in progress.

Learn more biomedcentral.com/submissions 